\title{
Intranet, una Experiencia Real en la Empresa
}

\author{
Amaia Arribas Urrutia \\ Departamento de Educación, \\ Universidades e Investigación del Gobierno Vasco
}

\begin{abstract}
Resumen: El objetivo de este texto es ofrecer un panorama sobre el uso de las redes locales computacionales en las empresas y el efecto en las mismas. El empleo de estas herramientas computacionales tiene como propósito mejorar la comunicación interna y reducir costes. Sin embargo, si esta tecnología no se vertebra en forma sistemática y estructurada con las estrategias de comunicación de cada organización, los objetivos de ésta, lejos de cumplirse, pueden verse retrasados o desviados. Nos encontramos, entonces, ante una nueva cultura de la gestión empresarial.

Abstract: The main objective of these text is to offer a general view about the uses and consecuences of local networks at the enterprises. The use of these informatic tools has the purpose of improve internal comunication and reduce expenses. However, if these technology is not well builded and sistematic structured, according with the comunication strategies of each organization, far away of reaching it goals, it could be delay or deflect. So we are now in front of a new entreprenurial culture.
\end{abstract}

la utilización de la tecnología de Internet para la generación, y
transmisión de información, así cómo para trabajo en grupo
sobre una red local, en el interior de la empresa se le denomina una Intranet. El responsable de red encuentra en una Intranet la utilización segura, por parte de una comunidad cerrada de usuarios, de las infraestructuras de telecomunicaciones de Internet con un objetivo de mejorar la comunicación interna y la reducción de costes.

La demanda de información de los diferentes agentes sociales ha transformado en pocos años la cantidad y la calidad de la información dentro de la empresa europea. Aunque se potencien unas facetas más que otras, la comunicación debe considerarse siempre de forma integral y de una forma estructurada y organizada. Es necesario que la comunicación -considerada como mera transferencia de información, lejos de definiciones complicadas- sea diseñada desde un punto de vista estratégico, puesto que en una organización, la comunicación interna proporciona una herramienta básica de gestión empresarial que puede ayudar a cumplir objetivos. 
No hay posibilidad de alcanzar una comunicación externa fluida si no existen vías de comunicación internas que están bien definidas por la propia empresa con el objetivo de motivar, integrar y desarrollar unos valores comunes entre todo el personal. Las personas que tradicionalmente han tenido poder en una empresa son las que saben dónde encontrar la información. Ellas serán las más afectadas por las nuevas tecnologías. El impacto más dramático para la empresa es el momento en el que se empieza a entregar informa- ción de forma diferente, es decir, a entregar nuevos caminos de acceso a la información. Según Jean-Louis Lezaun, director de Grandes Cuentas de MATROX, "dominar la información es una nueva apuesta para las empresas en su búsqueda de productividad" (Lezaun, 1997: 25-27).

La comunicación empresarial no debe ser neutra, ya que tiene que dar respuesta a unos objetivos concretos para conseguir determinadas metas empresariales. Además, para lograr esos objetivos se requiere una estrategia en materia de comunicación que cualquier empresa ha de pasar, en primer lugar, por asignar la responsabilidad de la comunicación, después definir la política de comunicación y crear una cultura comunicativa en el seno de la organización como instrumento de gestión empresarial dotándolas de los medios que le son necesarios para llevarlos a la práctica $\mathrm{y}$, por último, diseñar los canales y soportes de la comunicación.

La rapidez con la que las compañías han adoptado las nuevas tecnologías para su comunicación interna es vertiginosa. Si Internet se ha convertido en un verdadero modelo de sistema para publicar y compartir información, se ha estimado que el desarrollo de entornos Intranet llegará a ser el doble del crecimiento que ha experimentado Internet en los últimos tres años. Son un campo aún muy nuevo, pero su uso está creciendo aún más rápido que la propia Internet. Según estimaciones recientes, cada cuatro minutos se abre en algún lugar del mundo una instalación de este tipo, supone un nuevo concepto organizativo y de estructuración del flujo de trabajo dentro de las compañías (Sánchez, 1996). La mayoría de los especialistas en tecnologías de la información coinciden en señalar que las Intranets son un sistema revolucionario de presentación de la información; su compatibilidad con la mayoría de sistemas operativos, bases de datos e incluso programas contables, las convierte en una vía de acceso fácil a la información simultánea (Tuñon, 1997: 31-33). El resultado es que en 
la actualidad muchas empresas en Europa ya se han acogido a las ventajas que ofrece este sistema.

Y ¿cúal es la razón que ha empujado a la utilización de Intranets? La causa estriba, según Ignacio Martínez, de FUNDESCO, ${ }^{1}$ en la utilización de los mismos protocolos, del mismo software que Internet, $\mathrm{y}$ el hecho de que sea un entorno abierto en lo que respecta a su independencia de fabricantes y al tipo de ordenadores. La ventaja más sustancial radica en que todo tipo de línea física puede transmitir información con el Internet Protocol (IP).

En este artículo voy a analizar el uso de la tecnología Intranet por la empresa europea para establecer su comunicación interna, además de su estructura, seguridad, ventajas e inconvenientes de esta nueva forma de comunicación para la gestión y creación de la información en la institución empresarial.

\section{¿Qué son las Intranets?}

Las empresas que han ido entrando a la red $^{2}$ se han dado cuenta de un fenómeno, y es que Internet no sólo se emplea para acceder a recursos lejanos, sino que es una manera excelente de organizar los recursos internos. Se pueden tener páginas de Web privadas de la empresa para explicar la estrategia a seguir, o un servidor de FTP donde los

1 FUNDESCO (Fundación para el Desarrollo de la Función Social de las Comunicaciones) se funda en 1968 por Telefónica y patrocinada por diversas empresas del grupo. Juan de Vilallanga, presidente de Telefónica en España, preside el patronato. Su objetivo es analizar los efectos económicos de tecnologías de la información y la comunicación (TLC) y sus aplicaciones, realizando actividades que contribuyan a la plena integración de España y del mundo iberoamericano en la sociedad de la información.

2

El interés de las pymes españolas por las nuevas tecnologías aumenta poco a poco. Así, el 29,9\% estan conectadas a Internet o Infovía, cuando hace justamente un año ese porcentaje era del 18\%. Además, mientras en 1997 había un $13,3 \%$ de pymes no informatizadas, en 1998 ese porcentaje ha descendido al 12,3\%, según el Estudio sobre Equipamiemto Tecnológico de las Pymes, elaborado por CB Consulting para Microsoft y Telefónica. Otro estudio, el de Harris Research Europe, filial de Sofres, sitúa a los directivos españoles por encima de la media europea en la utilización de equipos informáticos en lo que se refiere a ordenadores de mesa (con un $88 \%$ ) y en cuanto al uso relacionado con las comunicaciones, como buzón de voz (69\%) y correo electrónico (79\%), en una encuesta realizada entre directivos empresariales europeos entre el 23 de septiembre y 31 de octubre del año pasado. 
empleados consulten todos los datos sobre la empresa, incluso empleen el correo electrónico para poner en contacto a colegas de delegaciones diferentes. Esta filosofía implica que la red local de la empresa ha de ser capaz de trabajar con TCP/IP.

Las Intranets constituyen la utilización de las herramientas propias de Internet y su aplicación directa al mundo empresarial. El Web como visualizador de la información, en combinación con correo electrónico, ftp, telnet, y el software de comunicación en tiempo real -IRC, InternetPhone, videoconferencia-, forman un conjunto de instrumentos con capacidad para crear una base sólida y estable, que cubre todas las necesidades de una empresa.

En un entorno cerrado y orientado hacia la empresa como es el caso de Intranet, los documentos corporativos pueden tener una apariencia uniforme a pesar de ser visualizados por personas que utilizan Macintosh o PC. Este hecho, junto con la simplificación de la confección de las páginas Web, significa que los documentos que tradicionalmente se han distribuido de forma impresa, puedan estar disponibles en línea y con acceso inmediato.

La estructura mínima que se requiere para instalar una intranet es una red de acceso local que soporte TCP/IP y la tecnología cliente/servidor. Si se tiene que intercambiar información con otras delegaciones de la empresa, se necesitan líneas de dedicación exclusiva, como las de punto a punto o Frame Relay. La rentabilidad de contratar una línea u otra depende del volumen de tráfico y de las horas conectadas diariamente. Los peligros del cliente-servidor clásico eran muy importantes y de consecuencias desastrosas. Durante la expansión cliente-servidor de los últimos años, las redes corporativas se han hecho más y más amorfas, extendiéndose hacia usuarios y clientes de todo el mundo. Esto ha convertido la gestión de recursos de SI en una tarea difícil, ya que los fallos de una aplicación pueden tener lugar en cualquier parte, desde el servidor de red hasta el escritorio de un usuario (Fryer, 1996: 38-42).

Cuando una organización plantea la posibilidad de implantar una Intranet, debería ser coherente con el resto de la estrategia corporativa respecto a Internet. Algunas de las decisiones que se habrán de tomar incluyen: qué aplicaciones se quieren implantar, qué calendario está previsto, cúal será la tecnología y cómo el personal de la empresa 
interactuará con el sistema, como simples usuarios pasivos, o como contribuidores de información y documentos.

Si se cuenta con una red de área local y se desea una intranet para gestionarla de forma totalmente privada, se pueden instalar servidores de Intranet que no estén conectados a Internet -denominados normalmente servidores off-line-. Esta es una opción bastante utilizada para consultar información en una Intranet, aunque los servidores de una Intranet suelen tener algún tipo de conexión permanente con Internet.

En una empresa con varias delegaciones, el sistema ideal es la implantación de servidores en cada delegación. La conexión se puede realizar fundamentalmente de dos formas: formando una red interna con la contratación de líneas privadas o bien contratando el acceso a Internet a través de algún proveedor de servicios o, en el caso de los servidores con firewalls (cortafuegos), directamente de la operadora. Un caso significativo es el de Cap Gemini, una gran empresa con delegaciones en el extranjero. La filial holandesa de Cap Gemini utilizó una Intranet para eliminar la montaña de informes sin leer y otros documentos. Ahora están almacenados en un servidor de la web y cualquier empleado accede a ellos utilizando un navegador estándar. Los empleados que trabajan fuera de su oficina acceden a los mismos documentos almacenados en la Intranet de la compañía utilizando un PC y un módem. El proyecto holandés es parte de una Intranet mayor, que pone en contacto a más de 17000 empleados y funciona sobre cuarenta servidores. Los empleados encuentran y recuperan rápidamente casos prácticos, diseñan documentos e informes sin importar dónde se encuentren.

Inclinarse por una u otra opción depende totalmente del estudio de la empresa. Para empresas de ámbito nacional y con un volumen importante de información, la solución más adecuada es la primera. En el caso de las empresas que cuentan con delegaciones en el extranjero, el elevado coste de las líneas internacionales hace más adecuada la conexión de todos sus servidores a través de Internet. ${ }^{3}$

3 La tarifa de conexión de todos los servidores de una empresa con delegaciones en el extranjero a través de una línea privada Frame Relay es de una cuota menusual y tarifa 
Este tipo de Intranets también puede resultar bastante rentables a las empresas cuyo volumen de tráfico sea reducido y que, por consiguiente, utilicen líneas punto a punto con módems de baja velocidad: con este tipo de líneas, estas empresas establecen llamadas locales para acceder a sus nodos de información.

\section{Estructura de la organización}

Los sistemas de información son un espejo de la organización. Si la organización crece de tal manera que las líneas de comunicación interna no funcionan, surgen relaciones alternativas de comunicación informal que no se adaptarían a la estructura deseada por la dirección.

Durante por lo menos tres décadas, se ha hablado en el mercado de la necesidad de que las organizaciones descentralicen la decisión de hacer y reestructurar el modelo tradicional de toma de decisión centralizado. Es ahora cuando la tecnología empieza a tener importancia a nivel de reducción de papel y de involucración de usuarios en actividades de trabajo en grupo. La comunicación electrónica permite superar muchas de las limitaciones de la distribución y de las modificaciones de los sistemas basados en papel.

El concepto de Intranet conlleva cada vez un mayor número de normas y herramientas que soportan las comunicaciones abiertas de red. Muchas de las herramientas que actualmente existen en el mercado se desarrollaron originalmente en entornos propietarios, modificándose para saltar al vagón Web.

Finalmente ha llegado la tecnología que permite a las empresas apoyarse en una toma de decisiones descentralizada y que coordina actividades. Su adaptación es fácil y barata para esas empresas que inicialmente investigaron sobre la arquitectura cliente-servidor, ya que ellas tenían la infraestructura técnica instalada. La tecnología Web

\footnotetext{
plana (depende de la velocidad de la línea elegida y de las necesidades de la empresa). El acceso a través de Internet por Infovía (Infovía es un servicio de Telefónica que proporciona la comunicación entre los usuarios y los proveedores de servicios de información con idénticas condiciones de coste para la comunicación con cualquier parte del mundo, marcando el 055 y sin necesidad de establecer un contrato con Telefónica.) es de 139 pts/hora. Esta segunda opción es siempre más barata que la anterior, aunque para la transmisión de grandes documentos se recomienda el alquiler de una línea Frame Relay.
} 
solamente proporciona el software para imponer una tendencia que ya había empezado a funcionar.

Internamente, muchas de las grandes empresas tienen los mismos problemas de información heterogénea y distribuida dentro de sus organizaciones que las tecnologías Internet y Web podrían resolver bajo ciertas condiciones. Y es fácil para muchas de estas organizaciones identificar los verdaderos beneficios al sustituir los procesos escritos por el flujo de información electrónica, ya que las redes son internas, las normas de seguridad y de acceso que se pueden imponer serían imposibles de aplicar sobre una red pública.

Estas empresas estudiaron cómo actualizar las anteriores tecnologías aplicadas a estos problemas corporativos, aplicando la tecnología Internet, pero dentro de un modelo cerrado. El modelo es limitado por diferentes razones, incluyendo seguridad, velocidad de líneas ...etc. Las tecnologías del software de trabajo en grupo más recientes han intentado proporcionar un enfoque distribuido tipo Internet.

La tecnología Web lo hace fácil y flexible para crear, publicar y acceder a la información, tan fácilmente que cualquiera con acceso a la base TCP/IP podría publicar dentro de la empresa, información que fuese accesible desde una gran variedad de plataformas cliente. Además, se pueden añadir rápida y fácilmente muchas aplicaciones de ayuda.

La flexibilidad y adaptabilidad de una Intranet hace que no sea necesario deshacerse de sus antiguos sistemas, sino que pueden seguir funcionando por debajo de la Intranet. El flujo de trabajo será una aplicación importantísima de las Intranets y ello no significa que se queden obsoletos los productos de groupware. De hecho, solamente el cuatro por ciento de los consultados, según la encuesta realizada por Datamation/Cowen a principios de este año, tenían la intención de sustituir su groupware, mientras que un treinta y cuatro por ciento aseguraba que iban a trabajar con el mismo software bajo el entorno intranet (Shamon, 1997: 41-42). El mayor desafío supone pasar de una actitud de control a una actitud que permita tomar decisiones y actuar de manera independiente.

La tecnología Intranet facilita las labores de creación y publicación de información dentro de la empresa. También facilita la recuperación y visualización de la información. Lo que ya no es una tarea tan fácil es 
averiguar qué información es realmente relevante dentro de este entorno independiente. Lo que falta en la tecnología Web es un canal de distribución y marketing para la información creada.

En varias empresas, la utilización de Web comenzó como una investigación realizada por el personal técnico, sin estar incluido dentro de la estrategia general y sin que se tuviera constancia de la importancia real que este proyecto traería consigo. A pesar de que muchos de estos proyectos son más conocidos en la actualidad que en sus comienzos, la mayoría se gestionan todavía separados del flujo principal del negocio.

La sobrecarga de información es una enfermedad de nuestro tiempo. Las tecnologías que en teoría iban a solucionar el problema sólo han servido para empeorarlo. Esto no es sorprendente si se miran todos los documentos que en formato papel se acumulan en el escritorio de cualquier oficina. Incluso, sin mencionar la cantidad de inevitable publicidad y papeles inservibles, la mayoría de la información que se envía a un trabajador sólo se envía por si pudiera necesitarla. Además de esta información, hay que añadir la que está desfasada, y la que no va a necesitar hasta un momento determinado. El profesional probablemente, archivará la mitad de la información enviada "por si acaso" y toda la información desfasada. Cuando vaya a necesitar la información, se encontrará con un alto volumen a tratar y con un sistema de gestión malo o inexistente.

La alternativa a la información enviada "por si acaso fuera necesaria" es la información enviada en tiempo real, o la información enviada cuando el profesional lo necesite. Esto ha constituido una promesa de los ordenadores y redes, que hasta ahora no había podido convertirse en realidad.

Históricamente ha habido dos enfoques principales respecto a la información enviada en tiempo real. El primero dejaba la información distribuida a través de las aplicaciones y sistemas. Para acceder a la información, el usuario debía conocer múltiples y complejos procedimientos de acceso. Una vez que se consiguiese llegar a las aplicaciones, cada una de ellas requeriría un interfase diferente. Los usuarios ignoraban la posibilidad de acceder a la información a tiempo real. A partir de la pasada década hemos avanzado hacia sistemas complejos que apoyan los sistemas de gestión. El cambio en las necesidades de la información, junto con las dificultades que ese 
cambio conlleva, han traído como consecuencia grandes sistemas gestionados de forma centralizada.

Para resolver este problema, algunas empresas intentaron reunir toda la información distribuida en un sistema maestro. Esto proporcionó al usuario la posibilidad de un acceso único y una sola interfase. Sin embargo, debido a que trataron de gestionar todos los requerimientos de la empresa de una manera centralizada, estos sistemas llegaron a ser lentos y complejos. Después de más de una década, todavía muchos sistemas no reúnen totalmente la información, porque el coste de la introducción de datos y su mantenimiento es demasiado alto.

La tecnología intranet ofrece un nuevo enfoque para obtener información en tiempo real. Debido a que da soporte a la creación de documentos electrónicos, la publicación y gestión de la información distribuida no requieren la complejidad de los antiguos sistemas centralizados. El documento es creado y gestionado por aquellos que lo originaron, sin tener que depender de los programadores para crear la entrada de datos.

Con los nuevos navegadores, un usuario puede recuperar y visualizar la información desde sistemas y fuentes distribuidos en distintos lugares, sin necesidad de saber nada sobre los servidores a los que acceden. Estos simples cambios en la tecnología revolucionarán las infraestructuras de información y cambiarán las empresas.

La característica clave de esta nueva tecnología es su capacidad para trasladar la capacidad de control de los flujos de información, desde sus creadores hasta sus usuarios finales. Si el usuario tiene la capacidad de recuperar y visualizar fácilmente la información cuando sea necesario, ya no hay necesidad de enviarla "por si acaso fuera necesario". Pueden separarse las publicaciones de la distribución automática. Esto se aplica a informes, normas, actas de reunión. La solución al problema es utilizar una Intranet para que la información pueda encontrarse y asimilarse eficientemente a través de peticiones.

El modelo de obtención de información tiene muchas ventajas. Las tecnologías tradicionales de transferencia de información están orientadas en lotes. Un lote de información se crea para ser entregado a través de documentos. Debido a que la información se empaqueta como un lote, cuando una parte cambia, no es frecuente que se actualice inmediatamente. Al ir pasando el tiempo, el lote va quedando 
desfasado. Para solucionar este problema, el lote entero debería abrirse cada vez que se dé un cambio. Entonces surgen varias cuestiones: ¿Cada cuánto tiempo puede permitirse la empresa la actualización de esos lotes y que coste conlleva?, ¿Cuánto tiempo utilizan los empleados en hacerla?

Como ya he mencionado antes, la tecnología Intranet apoya la creación y gestión de información distribuida, permitiendo la actualización de cada parte, al mismo tiempo que exige la mencionada modificación. Si se da la infraestructura adecuada puede accederse al contenido actual de la información fácilmente y encontrarlo cuando sea necesario. Esto reduce el tiempo necesario de formación y la cantidad de información que un empleado debe asimilar.

Existen varias razones por las que se decide instalar una Intranet en la empresa. No hay que olvidar que las necesidades de gestión, generación y organización de la información constituyen valores imprescindibles para la gestión empresarial, y que una red corporativa como Intranet lo ofrece de forma rápida, barata y fácil de utilizar.

\section{Gestión de la información}

Las organizaciones se autodefinen por la manera en que utilizan la información para coordinar sus actividades y lograr sus objetivos. Las tecnologías de gestión y distribución de la información han cambiado a lo largo del tiempo. La información de la organización generalmente contiene datos que sirven para tomar decisiones que pueden ocasionar pérdidas o ganancias de recursos. Al implantar la tecnología Intranet, una compañía aumenta su capacidad para controlar los recursos de los que dispone. Las actividades y sus progresos deben estar coordinados para que la compañía saque adelante sus objetivos. Con el fin de alcanzar dichos objetivos, se tiene que estudiar cómo se genera la información de la organización, cómo se distribuye y cómo se aprovecha. Por ejemplo, no sería muy eficiente si un individuo necesita determinada información y desconoce su existencia o su ubicación.

Alguna información se actualiza constantemente y la mayoría de las empresas hasta ahora lo han hecho en formato papel. Algunas empresas son relativamente estables en su estructura y actividad. En este tipo de casos la actualización de la información es más sencilla, ya que su contenido puede no cambiar aunque las actividades no están coordinadas, sean extensas y complejas, y la información se recoja de 
una forma centralizada y posteriormente se distribuya. Sin embargo, no es normal que se den estas características hoy en día en la mayoría de las empresas.

\section{La generación de la información}

La documentación que se publica en una Intranet surge de diferentes fuentes y de diferentes maneras: anuncios o información oficial de la casa, emitida y aprobada por la dirección; información que generan los empleados o información suelta que generan individuos y que consideran de interés general para la casa, por ejemplo, pequeños anuncios, sugerencias de mejora, tablones electrónicos de anuncios, etcétera.

En el caso de información generada a nivel departamental, su objetivo no es estratégico, como en el caso de la información oficial, sino el de compartir noticias e ideas de interés para el resto de la organización y que podrían contribuir a la actividad de otra área. Según el grado de confidencialidad de dicha información, es necesario prestar cierta dedicación - por parte del departamento que la genere- a definir derechos de acceso para esta información.

Muchas empresas han descubierto que la posibilidad de publicar información en una Intranet, da lugar a una inicial actividad frenética cuando los empleados ven que es fácil dejar en la red corporativa información que otros usuarios aprovechan. Una Intranet en su fase inicial se llena rápidamente de información. Normalmente un directorio personal en un servidor Intranet sirve como un depósito de papeles, notas y conceptos que comparten miembros de la empresa, cuyos intereses sean comunes para la solicitud de comentarios o por cualquier otro motivo. En vez de hacer copias, las partes interesadas abren la pantalla en cuestión, y pueden leer e investigar la última versión mientras se producen los cambios. Este tipo de información informal se convierte en un estímulo muy grande para el desarrollo de nuevos conceptos e ideas.

\section{La organización de la información}

Tan importante es la organización del contenido de una Intranet que se hace necesaria la creación de índices y directorios que aparecen en páginas de orientación. Si las páginas de contenido tienen información de valor pedida por el usuario; son estas páginas las que ayudan al 
usuario a encontrar las páginas de contenido apropiadas para sus necesidades en cada momento.

Los documentos que se publican en una Intranet tienen diferentes características; contienen información que no se modifica o son páginas activas en las que el contenido de la página se genera sobre la marcha, desde una base de datos u otro centro de información. Incluso las páginas más estáticas se convierten en puntos de información interactivos con la introducción de nuevas tecnologías, como las multimedia.

En cuanto a las páginas que contienen los índices y los directorios, utilizan también diferentes tecnologías, pero todas tienen la misma función: ayudar a los usuarios a encontrar información relevante. Las buenas páginas de orientación sirven para una audiencia definida. Además de los nexos que contienen con otras páginas, también tienen una pequeña descripción del contenido al cual se refieren, para que el usuario evalúe la utilidad del documento.

Otra funcionalidad importante que las páginas de orientación deben ofrecer son los programas de búsqueda de contenido con conjuntos amplios de claves de búsqueda. Como en el caso de programas de búsqueda en el entorno abierto de Internet como Altavista, cuando el número de documentos relacionados con un término o clave de búsqueda es tan grande que el usuario no puede revisar todo, el sistema valora y ordena los documentos en función de su grado de relación con las palabras buscadas.

\section{Uso de la Intranet}

La publicación de documentos internos es el uso más frecuente de las Intranets, permitiendo al personal el rápido acceso a determinadas informaciones. Por ejemplo, el área de recursos humanos utiliza una Intranet para publicar documentos - boletines informativos, calendario laboral, cursos de formación, entre otros-. O el departamento de marketing pone en una Intranet sus próximas campañas orientadas a informar al propio personal de la empresa antes que al público (Edmondson, 1996: 49).

¿Pero para qué sirve una Intranet? Las propiedades de la tecnología Web aplicadas a las Intranets nos permiten gestionar de una forma sencilla el trabajo. Por ejemplo, al trabajar con una base de datos de una Intranet consultamos y modificamos una determinada información 
desde cualquier oficina de la empresa, mediante formularios editados en HTML. Naturalmente para evitar la actualización on-line simultánea desde distintos puntos - que sería caótica-, se establecen en su diseño restricciones de acceso mediante contraseña y privilegios de lectura y/o escritura dependiendo del usuario.

Otra de las posibilidades de las Intranets es la edición de todo tipo de manuales, guías, instructivos y otros documentos. También se editan digitalmente las revistas o las notas de prensa dirigidas a los medios de comunicación. Al permanecer en la red se ahorra un gasto innecesario de papel y fotocopias, evitando la publicidad y réplica de los documentos de las distintas sucursales. El empleado accede a la red para consulta o para impresión. Las intranets se convierten en tablones de anuncios para la publicación de notas internas o para crear catálogos, para incluir características técnicas de los productos y visitas virtuales a la empresa.

Diseños con nuevas tecnologías como el VRML permiten visualizar en 3-D diseños industriales o el plano virtual de la empresa, mediante el cual accede a más información haciendo click con el ratón en el edificio que nos interese.

Los usos internos más frecuentes en una intranet son (Expansión, 12-7-1996: 7) ${ }^{4}$ :

1. Información publicada

- Información estática.

- Procedimientos y normas corporativos.

- Información de clientes y productos.

- Información de recursos humanos y listados de tareas.

- Materiales de formación.

- Materiales de investigación y acceso al sistema documental.

- Mapas y direcciones.

- Organización de estructuras y datos de empleados.

- Información que aporta un ámbito específico.

- Información financiera de la empresa.

${ }^{4}$ El autor cita como fuente a Microsoft.(Nota del editor) 
- Plantillas corporativas.

- Inventario en tiempo real o información de ventas.

- Planificación de datos.

- Investigación de datos.

2. Trabajo en grupo y fluido

- Proceso presupuestario.

- Tablones de anuncios.

- Diseño de ingeniería y fabricación.

- Comunicaciones de grupo a través de correo electrónico.

- Previsiones de grupos, incluyendo usuarios móviles.

- Planificación de recursos humanos.

- Planes de marketing.

- Procesos de informes de gastos

3. Procesos de negocio interactivos

- Comercio electrónico.

- Enlaces entre los sistemas de colaboradores.

- Servicios de atención al cliente.

- Sistema de inventario de fabricación.

\section{Estructura de una Intranet}

La estructura de información en una Intranet es determinante para la facilidad de gestión y la efectividad del sistema. Dicha estructura, desde un punto de vista global, representa el mapa de la información de la empresa. El mapa permite ir directamente a los documentos o datos que uno busca, a través de los nexos en los documentos.

Una página de mapa de la información consiste únicamente en nexos, salvo cuando se mezcla con páginas de contenido. Esto no es recomendable, ya que el mantenimiento de ambos tipos de páginas se combina y puede dar lugar a errores de acceso. Cada página mapa debe corresponder a un nivel en la estructura organizativa de la empresa, cada uno con su zona de información. En las organizaciones actuales se tiende a jerarquizar, centralizar y guardar celosamente la información, pero la técnica de mapas se aplica a la mayoría de los modelos organizativos. 
En vez de reflejar la organización o las funciones, el mapa es un mapa lógico donde el nivel superior es el objetivo, el siguiente nivel abarca los principales enfoques requeridos para alcanzar el objetivo, $\mathrm{y}$ así, hasta el nivel inferior del contenido.

Independientemente de quién sea el responsable de la página mapa global, la finalidad de la misma será servir de nexo entre las páginas mapa. La persona responsable modifica cada página e introduce la información que genere su área aunque la identificación del material sea una responsabilidad común para todos los empleados del área.

No obstante, cada organización decide la política puntual que se aplica a sus necesidades. Normalmente, organizaciones más pequeñas y con menos imagen pública tienen políticas más flexibles en cuanto a la gestión de la documentación en sus Intranets. En el caso de la administración, por ejemplo, el asunto se complica por cuestiones de volumen.

El mapa proporciona una visión global de la interrelación de todos los componentes de la organización, por lo menos desde el punto de vista de su contribución de información y documentos. Un usuario conoce las relaciones entre departamentos al comenzar en un punto de mapa y seguir los nexos a todo el contenido que apoya las funciones para las que son responsables. Un mapa de la información revela mucho sobre la organización. Cuando ya está implantada la Intranet, los departamentos empiezan a cargar documentos en sus zonas correspondientes, mostrando la información una estructura lógica. Cuando hay una reorganización, los gerentes deben ajustar el mapa rápidamente, cambiando uno de sus nexos, y el contenido no necesita moverse.

Una gran ventaja de la arquitectura de estos mapas es su flexibilidad. Se puede originar de arriba a abajo o de abajo a arriba. Si muchas delegaciones desarrollan sus Intranets independientemente, con este tipo de estructura de mapa de negocio, podrán unirse en el futuro. Esta flexibilidad es el resultado del modelo de coordinación central de toma de decisiones distribuidas.

En las instalaciones Intranet que permiten la publicación de información no oficial, como es el caso de un tablón de anuncios, un mapa de la información no sirve de mucho, ya que la información no se conforma con ninguna estructura relacionada con la empresa. También está el caso de las páginas en curso, cuyo contenido formal aún no ha 
sido finalizado. Para ello debe hacerse un mapa privado que funciona de la misma manera que el mapa de la empresa, pero no es públicamente conocido y se protege mediante passwords u otros controles de acceso.

El mapa privado se mejora con algunas páginas de nexo adicionales. En este modelo, el autor mantiene una página índice que se divide en dos secciones, la del trabajo en curso y la del trabajo acabado. Cuando se crea el trabajo en curso, al autor lo sitúa en el directorio Web y lo vincula con la página Index. Mientras se van haciendo las revisiones, el autor sitúa las últimas versiones en el mismo directorio con el mismo nombre, de tal manera que el índice se direcciona automáticamente hacia la última versión. Esto no excluye que se guarden versiones previas si es necesario. Las versiones previas se copian, se numeran y se sacan de la versión actualizada. Revisado el contenido, va a producción y el autor saca de la parte del trabajo en curso y redirecciona el nexo a la sección de trabajo terminado.

En las organizaciones de servicios y proyectos, un cierto tipo de directorio que es de interés es una página de proyectos. El jefe de proyecto crea esta página que contiene líneas de nexos para todos los proyectos parciales. Cuando el autor crea el primer borrador, no sólo se anexa el contenido del archivo, sino que también notifica al jefe de proyectos su localización para que el contenido se una a la página de proyectos. Esto proporciona una rápida trayectoria de trabajo, tanto de los trabajos en curso como de los finalizados para todos los empleados. De nuevo, una vez que las estructura está en funcionamiento, requiere poco mantenimiento de la información actualizada.

\section{Métodos de búsqueda de información}

Las páginas de orientación son la forma principal para que los usuarios encuentren la información en una Intranet. El mecanismo que facilita ir a la información, son los nexos. Los nexos guardan grandes áreas de contenido, con material relevante de gran número de empleados, para que éstos no dupliquen el proceso. Se identifica la información oficial y proporcionan interpretación de la información general en el contexto de la organización.

La mayoría de las organizaciones también tienen páginas de nexo de tipo informal que sirven de páginas de orientación. Un individuo comienza una página por sí mismo; sin embargo, hasta que estas 
páginas no estén en una estructura de compromiso formal, no hay garantía de que el autor mantenga el contenido actualizado.

El directorio informativo de una empresa, a veces denominado páginas amarillas, organizado por materias, ayuda a los usuarios a encontrar el nexo. El mantenimiento del directorio informativo se lleva a cabo como norma general por el administrador Web o el experto en Web de la empresa. Debido a la incorporación de las páginas de orientación de tipo informal en un directorio informativo de empresa, es necesario incluir algunos mecanismos nuevos para que el directorio no se llene con páginas desfasadas o abandonadas. El reto consiste en proporcionar una visión centralizada sin imponer una centralización que provocaría muchísimos atascos.

Una forma de manejarlo consiste en crear una caducidad automática para todas las páginas que no gestionan oficialmente la entidad de la organización. Cualquier responsable de sitio lista sus páginas enviando un formulario Web o correo electrónico al administrador Web. Sin embargo, las páginas informales sólo se incluyen en el directorio durante un mes (por poner un plazo). Si el responsable no renueva la petición en esos sesenta días la página será eliminada del directorio. Permite que las páginas informales se publiquen y evita que el directorio se convierta en un depósito de vínculos a páginas abandonadas.

El índice de la empresa proporciona a los usuarios otro método de búsqueda de información, relacionado frecuentemente con el funcionamiento de búsqueda. Dicho índice y la función de búsqueda no necesitan que sus páginas se relacionen con el resto de las páginas de orientación o que se gestionen por un software específico. Un agente organiza el programa de búsqueda y el índice, un programa que regularmente investiga la Intranet y cataloga el contenido. Este es otro medio disponible para que los usuarios encuentren el contenido que buscan.

\section{Control de la información corporativa}

Los usuarios acceden y visualizan la información. Hay muchos motivos por los que un usuario accede a la información, y estos motivos, probablemente, variarán entre una sesión y otra. El usuario debe tener en cuenta dónde se crea el valor. Es decir, si la dificultad para acceder a la información excede del valor que la información tiene 
para ellos, o bien no utilizarán esa información u otros medios para conseguirla.

Los departamentos crean información. En los medios tradicionales, la creación de documentos y la estrucutra están estrechamente ligados. Las trayectorias de acceso son principalmente lineales y toda la información relacionada se une y se entrega unida. Si se necesita un camino de acceso diferente o una combinación de información, es más eficiente implantar la estructura de un documento nuevo, duplicando la información común y utilizando índices externos que unan las diferentes partes de los distintos documentos.

En el nuevo mundo de los nexos múltiples, en los hyperlinks, el aspecto estructural seguido en la creación del documento cambia. Varios factores conducen a ello:

1. Los hyperlinks permiten al usuario escoger el orden en el que quiere acceder la información. Aunque el autor continúe necesariamente proporcionando la estructura, la función de esa estructura cambiará al determinar a priori cúales son las necesidades de los usuarios, ayudándoles.

2. Cuando la información se combina de un modo distinto, el original se une por nexos múltiples. Esto tiene un impacto significativo sobre la creación de documentos Intranet. El objetivo consiste en reunificar la información más que en mantener copias separadas que se sincronizan continuamente. Los autores están más interesados en estructurar la información en módulos reutilizables y se olvidan de crear estructuras lineales específicas.

Los propietarios de información son clave para organizar y encontrar la información. Esto es cierto en el mundo del papel y seguirá siéndolo con la tecnología Web. Pero la diferencia en un entorno Intranet es que los conocimentos sobre la ubicación e importancia de la información se hacen más tangibles en forma de directorios e índices de acceso.

Una guía de empresa en el pasado era un documento de 300 páginas. Con la tecnología Web es una única página de hyperlinks. Además hay que tener en cuenta el enorme esfuerzo de los empleados al cortar y pegar para crear un solo documento, mientras que con la tecnología 
Web se sustituye el tiempo y el esfuerzo para unir y publicar texto con líneas únicas, uniéndolas por nexos múltiples al texto original.

En cuanto a las herramientas de editor, llegamos a conocer el verdadero valor de una Intranet, cuando las diversas partes participantes aportan algo a su contenido. Para conseguir esto, no es necesario que los individuos aprendan lenguajes de programación especiales ni dominen el funcionamiento de software. En cambio, debe haber herramientas disponibles que permitan enviar información sin más dificultad que la de utilizar un programa estándar de procesamiento de textos.

No es necesario que las Intranets sean demasiado exóticas o que estén llenas de aplicaciones ultramodernas. En la mayoría de los casos, las interfases y aplicaciones más simples son las mejores. La clave es que el sitio refleje el modelo de negocio de la empresa, y que refuerce el sentido de comunidad entre los empleados de la empresa.

La creación, gestión y divulgación de la información son procesos que no se gestionan correctamente en la mayoría de las empresas. Normalmente se deja que los grupos y organizaciones internas implementen sus propias soluciones sin una metodología común, lo que crea soluciones incompatibles.

Las empresas usan múltiples métodos para la gestión de documentos. Estos métodos varían desde archivar documentación impresa hasta usar un recurso compartido de red. En la mayoría de los casos, las gestiones del funcionamiento y el contenido del documento son locales a individuos o a grupos. Ninguna de las soluciones actuales es factible para ser implementada en una empresa en su conjunto. Por lo tanto, algunos grupos no pueden acceder a la información que necesitan fácilmente.

Muchas empresas han comenzado a usar tecnologías WWW para resolver estos puntos. Como muchas compañías adoptan tecnologías WWW a un nivel interno, tienden a concentrarse en la pura difusión de la información, lo que incorpora conversión de documentos, y su edición junto con los procesos de recuperación. Normalmente la conversión y la edición de documentos son responsabilidad de las personas que trabajan en el departamento de los sistemas de información. Muchos sitios Web internos comenzaron como proyectos informales de un área técnica donde el grupo que lo formaba se responsabilizaba de convertir documentos corporativos y publicarlos 
en la Web interna para conseguir compras por parte del resto de la empresa.

\section{Seguridad en una Intranet}

El mundo de los negocios tiene cada vez más información para gestionar. El resultado de la explosión de conectividad que se está experimentando en nuestros días se traduce en un enorme flujo de información que, paralelamente, aumenta la vulnerabilidad de la misma. El valor de la información es incalculable y, por tanto, debe protegerse (Huidobro, 1996: 54).

Se plantea a muchos directivos la posibilidad de configurar un servidor Web de modo que no sea parte de la Intranet. Es posible, pero habría que cargar la información en la máquina manualmente. Además, quien acceda a este servidor Web desde Internet se queda allí, sin poder avanzar más. Esta configuración es buena para sitios Web que únicamente van dirigidos al público: aquellos que llevan información sobre el producto, historia de la empresa, direcciones ...etc. Pero esta configuración no es de utilidad alguna para los trabajadores que lo hacen desde casa.

La experiencia demuestra que los sistemas con configuración independiente no continúan siendo independientes durante mucho tiempo. No es muy práctico cargar la información manualmente, y representa un avance muy pobre en la aplicación de la tecnología de la información. El método más práctico y seguro de conectarse es a través de Internet, eso sí, utilizando los cortafuegos.

Un firewall es un mecanismo que permite controlar el acceso a usuarios a ciertas zonas de una red. En el contexto de las Intranets, se suele emplear como medida de protección, de manera que los usuarios externos vean sólo una parte reducida de la Intranet, concretamente aquella designada como pública. Es frecuente encontrar firewalls que actúan a modo de filtros de acceso. Cada vez que un usuario conecta con la red, se mira su dirección, y se decide si se le deja pasar o no. Generalmente, los firewalls suelen interponerse entre el servidor web y la red interna, que debe ser confidencial".( Expansión, 12-7-1996: 7)

Las Intranets que utilizan redes públicas tales como Internet se denominan "redes privadas virtuales" o VPNs. Por ejemplo, ADEGI

5 El autor cita como fuente a Microsoft. (Nota del editor) 
(Asociación de Empresarios de Guipúzcoa) tras un año de pruebas piloto tienen acceso directo -con clave de red interna y de correo electrónico- a una Intranet privada en Internet. Los empresarios de ADEGI pueden encontrar en AdegiNet, mediante un sistema sencillo de búsqueda, todas las circulares, información de ayuda actualizada, boletines oficiales, convenios vigentes, concursos, etc. ${ }^{6}$ También el Colegio Oficial de Ingenieros de Bizkaia ofrece una Intranet a través de su página en la Web. Sus colegiados pueden encontrar en la red de acceso público información sobre cursos, becas, la revista Dyna (en construcción), proyectos, una visita guiada por el Colegio y enlaces de interés, pero necesitan un password para acceder a la base de datos que le ofrece su Intranet.

La red presenta riesgos respecto a la inviolabilidad de sus múltiples puntos de acceso, la integridad de la información que circula por ella o la autentificación de los usuarios. Para evitar estos problemas, se requiere un modelo de seguridad que garantice el secreto de las comunicaciones, impida accesos no autorizados, asegure la correcta utilización de los usuarios y consiga la integridad de los mensajes. Los usuarios reclaman soluciones integradas para evitar violaciones en la seguridad de sus redes. De ser posible, un mismo sistema a través del cual puedan manejar toda la network y en el que se incluyan las aplicaciones más fiables para conseguir una comunicación segura, a través de las redes corporativas: cortafuegos que impidan accesos no autorizados, sistemas de escáner y auditoría que controlen e informen cualquier actividad sospechosa en la red, sistemas de encriptación, sistemas de autenticación de usuarios y sistemas de control de acceso a la red (Molina, 1997: 38).

Sin embargo, la seguridad es aún una asignatura pendiente para las Intranets. Los requerimientos de seguridad para poder establecer una red segura son:

6 El Servicio Empresarial de Comunicación Integral AdegiNet es un instrumento a disposición de las empresas guipuzcoanas que ofrece disponer a tiempo real de toda la información relativa a ayudas, concursos públicos, subastas, ferias. Además, ADEGI es la puerta de entrada de estas empresas a la red Internet al ser proveedor de acceso. Una opción que permite investigar en Internet, además de las secciones que ADEGI dispone de su propia página en la Web. 
- Confidencialidad: garantizar que los datos no sean comunicados incorrectamente.

- Integridad: proteger los datos para evitar posibles corrupciones o cambios no autorizados.

- Autentificación: tener confianza en la identidad de los usuarios, servidores y clientes.

- Verificación: comprobar que los mecanismos de seguridad son sólidos, potentes y que están correctamente implementados.

- Disponibilidad: garantizar que los recursos estén disponibles cuando se necesiten.

Los peligros del desarrollo de una Intranet son importantes. Todas las empresas que plantean un proyecto Intranet estudian de forma detallada y seriamente el concepto de seguridad con mayor cuidado incluso que dentro de un proyecto de Internet. Y con más cuidado aún si la Intranet plantea el uso del transporte público Internet como vía de acceso."Un cortafuegos hace mucho más que proteger simplemente la red de posibles intrusos. Puede proporcionar también información vital sobre la actividad del sistema".(Lefevre, 1997)

La seguridad se estudiará desde tres perspectivas fundamentales:

- El acceso a la información (quién y bajo qué circunstancias accede a la información disponible en Intranet). La encriptación de los datos (prevención ante un posible pinchazo de la línea). La defensa contra los ataques desde el exterior (el firewall pierde su efectividad cuando un acceso exterior puede entrar en la base de datos corporativa.

- El propio diseño de la aplicación. Una mala planificación de accesos, el mantenimiento y frescura de la información suministrada puede dar al traste con la misma.

- La propia velocidad de aparición de novedades en el mercado. "Resulta difícil establecer estándares (...) cuando un día y otro también surgen novedades no sólo en productos sino en posibilidades que sobrepasan las previsiones de los analistas informáticos que desarrollan las aplicaciones" (Cruz, 1997: 38).

Existen diferentes métodos de seguridad (más de 150 diferentes) que, combinados, foman un sistema cerrado, sin posibilidad de ser visto desde la red aunque se esté conectado físicamente a ella. Sus 
cometidos son esencialmente los mismos: detienen cada llamada, la vetan y/o la dejan pasar sólo si cumple con requisitos preestablecidos.

Controlamos el acceso a una red interna mediante la autentificación de contraseñas. Una vez que cada delegación posee una dirección IP fija, se administra el permiso de acceso a las direcciones conocidas. Existen cortafuegos que por defecto poseen métodos de encriptación de la información -según los protocolos internacionales de seguridadque codifican la información al ser transmitida de un lugar a otro de la red.

Pero, ¿cuántas barreras de protección se necesitan para que sean realmente efectivas? Depende de si se quiere dividir la red interna en diferentes compartimentos estancos protegiendo cada uno con una barrera de protección, según las necesidades de cada uno. Pero existen limitaciones a las barreras de protección como son los virus y el hecho de que sea muy protectora, impidiendo el acceso a algunos servicios deseables para la empresa. Tampoco previene de los problemas en el interior de la red, como los cuellos de botella debido a un alto volumen de tráfico o cuando se utilizan programas anti-virus. Además, es un punto simple de fallo, y si algo se estropea, nada podrá entrar ni salir.

\section{Ventajas e inconvenientes}

Internet es una herramienta muy útil; que ayuda a una mejor y más rápida realización de las tareas. Una Intranet resuelve diferentes problemas de comunicación interna de las empresas. Esta mejora se deja notar especialmente en dos áreas, que son la mejora de la comunicación entre los empleados o departamentos y la mejor estructuración de la información interna.

El hecho de contar con un protocolo como TCP/IP nos permite usar el tan popular correo electrónico. Avisos, circulares, notas, informes todo se puede enviar por correo, con un considerable ahorro de tiempo y dinero. Por otra parte, gracias a su velocidad, los empleados mantienen en contacto casi continuo y mejoran su rendimiento.

Cuando se habla de estructurar la información, nos podemos imaginar un típico día de trabajo en los departamentos de la empresa: docenas de empleados transfiriéndose gráficos, dossieres, documentos; en resumen, información. El flujo de datos es unidireccional, y esto es uno de los grandes problemas que las Intranets resuelven. Dejándolo en un servidor Web nada se pierde, y todo estará 
bien organizado. Son muchas las empresas que ya disponen de homepages de sus diversos departamentos, cada una con los datos correspondientes a su área de trabajo. Además de un considerable ahorro en papel -que puede ser más importante de lo que parecemejora la eficiencia. En la empresa tradicional, cuando algún empleado pierde un informe tarda varias horas o incluso días en tener uno nuevo disponible. Gracias a las Intranets se garantiza que el documento estará en su pantalla en menos de cinco minutos (Sánchez, 1996).

En primer lugar, existen ventajas económicas concretas derivadas de la integración de las Intranets en la empresa, como es la publicación barata y ecológica de documentación interna, y una funcionalidad que todavía se está asentando en las empresas como es su utilización como una herramienta de trabajo en grupo y de control de flujos de trabajo. Las ventajas que ofrece una Intranet también las tienen otros productos en el mercado, pero los productos de Intranet proporcionan una interfaz que reduce las necesidades de formación, y por lo tanto sus costes.

Pero, ¿cuánto cuesta una Intranet? Primero, hay que conocer el coste de la actual red cerrada interna. Si la empresa está localizada en diferentes lugares, habrá que alquilar líneas telefónicas y otro material de redes de empresas especializadas como es una compañía de transmisión de datos. No se necesita una red de altas prestaciones entre cada punto de la organización. En el nivel más simple, se crea una "red privada virtual" (significa lo mismo que una Intranet) con un módem en cada edificio. Los mensajes de correo electrónico se envían en grupo cada 10 minutos a través de un ordenador servidor configurado para este propósito. Esto, por supuesto, es una Intranet en su forma más simple. Pero demuestra que se pueden satisfacer las necesidades de la empresa según el precio que se esté dispuesto a pagar.

Resulta muy fácil para los usuarios localizar documentos que necesiten mediante la incorporación de menús y funciones de búsqueda. Los productos Intranet son abiertos en el sentido de que sus estándares son de dominio público y permiten un grado de flexibilidad mucho más alto del que estaba disponible anteriormente, haciendo mucho más atractiva a los programadores la idea de diseñar productos que mejoren las características de Internet $\mathrm{y}$, por lo tanto, de los 
entornos Intranet. Esto da lugar a que una Intranet sea más barata de implantar y mantener.

La calidad de la información en una Intranet se controla a través de diferentes mecanismos, incluyendo el control de versión de documentos, el mantenimiento de esquemas de planificación, la realización de estadísticas de uso (quién lee, qué, cuándo y con cuánta frecuencia), y además de controles de seguridad para controlar los derechos de acceso y manipulación de la información.

Los beneficios clave de un sistema de este tipo incluyen una mayor comunicación entre los empleados servida por una Intranet, la posibilidad de que la información sea mantenida por su propio propietario y que esta área de la organización participe en decidir sobre cómo se divulgarán los documentos, la conservación y organización de información, la disminución del tiempo para encontrar determinada información y el apoyo para las soluciones desarrolladas rápidamente.

Los ahorros de coste incluyen la reducción o eliminación en el proceso de gestión actual de documentos, la reducción o eliminación de producción y difusión de materiales de documentación impresa, la reducción o eliminación de reproducción de documentación perdida, y el aumento en el rendimiento y la optimización en la toma de decisiones realizadas por los empleados bien informados.

Por su naturaleza, las Intranets tienen gran capacidad de crecimiento. Crecen y evolucionan al mismo tiempo que la empresa. Se empieza con pocos usuarios y un único servidor Intranet, y llega a tener una amplia red para toda la compañía. Cuanto más crecen las Webs internas y más aumenta la demanda de conversión y edición de documentos, más necesarios son los métodos que permiten a los propietarios de la información publicar y mantener sus propios documentos en la Intranet. Además, los métodos para la búsqueda y la recuperación de los documentos necesarios son cada vez más importantes para los usuarios de una Intranet.

Son más ventajas que inconvenientes los que ofrece esta nueva tecnología. Resumimos, por tanto, las ventajas que la red privada interna ofrece:

1. Los Webs internos permiten a los empleados buscar información interna-clientes, su perfil, políticas de empresa, etc.- así como 
información generalmente escondida en base de datos y cuyo acceso resulta muy complicado.

2. Mediante un navegador, es posible acceder a la información sin que sea necesario tener en cuenta la plataforma en la que se encuentra, el tipo de ordenador o el tipo del sistema operativo.

3. Las Intranets conectan los distintos tipos de ordenadores, ubicados en diferentes delegaciones.

4. Estos sistemas de información corporativos sólo necesitan, en materia de software, un programa navegador y un programa servidor en el que reside y se organiza la información.

5. Intranet se basa en una tecnología barata en comparación con otros sistemas de información corporativos ya que, en otros aspectos, su sistema de correo electrónico permite la coexistencia de soluciones de distintos fabricantes. Por lo tanto, se eliminan los costes de licencias de correo electrónico y su multiplicación con el crecimiento del número de usuarios. Además, conecta vía telefónica puntos de todo el mundo al mismo precio de una llamada local.

6. Las redes privadas vía Internet se benefician de un elevado nivel de seguridad. Esto es debido a que están protegidas por un software de firewalls, un password y otros sistemas de identificación.

7. Uno de los principales y más importantes pilares de la competitividad futura dentro del entorno empresarial se encuentra en las grandes y variadas posibilidades que puede llegar a ofrecer la red, en especial mediante la utilización de tecnologías que están basadas en Web para aplicaciones internas.

Cada vez hay más documentación sobre el impacto que las Intranets tienen sobre la productividad corporativa y sus costes. Sin embargo, el verdadero potencial de los sistemas de información abiertos reside en integrar la empresa virtual por medio de Intranets que se extienden más allá del entorno corporativo. Estas Intranets extendidas ofrecen el potencial para resolver muchos de los problemas de compatibilidad y de compartir informaciones que se dan en una estructura de plataformas cruzadas. 
Las Intranets extendidas ayudan a las empresas a realizar muchas de las actividades más esenciales y costosas con las que un negocio debe lidiar. Compartir y distribuir el contenido de la información ha llegado a ser una de las aplicaciones dominantes en la Web.

Las compañías y editores en los Estados Unidos, producen diariamente tres billones de páginas de información, destinada a informar a los socios y empleados de la compañía. Pero debido al alto coste y a la cantidad de tiempo que esta labor requiere, normalmente se consigue el efecto contrario (materiales anticuados y mal preparados).

Para crear expectativa de crecimiento y que pique la curiosidad del usuario para que vuelva a visitar su sitio, algunos webmasters envían boletines periódicos por correo electrónico en los que informan sobre los cambios producidos en los sitios. Luego, esos boletines pueden situarse en archivos de búsqueda de hipertexto.

Un acceso bien diseñado permite a sus usuarios encontrar la información rápidamente. Lo que la gente espera de su sitio Web es encontrar la información de un modo fácil y rápido. La hospitalidad es la clave; los sitios Web que son dignos de volverse a visitar están muy restringidos. Esto significa que no debería añadirse sonido, video o animación al sitio Web simplemente porque se disponga de medios. Se debería evitar el color gratuito, mantener las páginas en un tamaño razonable y vincularse con otros sitios mediante nexos.

La eficiencia es la otra cara de la moneda en cuanto al acceso, y es más útil al propietario del sitio que a sus usuarios. Su sitio de Web Intranet debería estar estructurado de tal manera que su mantenimiento resulte sencillo.

\section{Conclusiones}

Desde hace dos años, coincidiendo con el inicio del éxito de la WWW, las empresas europeas vienen usando la tecnología Internet. Se han dado cuenta de que es una alternativa para desarrollar redes internas que aumentan el rendimiento en la gestión de grandes volúmenes de información; por tanto, la Intranet juega un papel estratégico en la empresa.

Internet e Intranet modifican el modelo de empresa en Europa. Internet revoluciona las comunicaciones, y su penetración en el ámbito empresarial da lugar a drásticos cambios en la distribución de la información. Las Intranets se convierten así en un mecanismo eficaz 
para compartir información, e integran facilidades de creación, mantenimiento, uso y acceso. La eficacia en la distribución de la información es un factor destacable de las mismas, la disponibilidad de datos es inmediata, y la actitud del usuario es activa. La Intranet destaca, entre otras cosas, por su capacidad para transformar al usuario de una estación de trabajo en un miembro activo de una red cooperativa, ya que las oportunidades que ofrece la red son tremendas: cooperación, distribución, acceso a la información, etc. Así el usuario tiene un mayor protagonismo.

Una Intranet presenta una serie de oportunidades muy interesantes para alcanzar propósitos de comunicación de carácter estratégico y táctico. El hecho de que las redes internas ayuden a potenciar el trabajo de sus empleados, reduciendo el tiempo que se tarda en gestionar la información y aumentando el flujo de datos, supone una mayor ventaja competitiva para la compañía.

Establecer una Intranet supone hablar de un potente sistema de comunicación, de reducción de costes, de mayor calidad, de mejorar la relación con los empleados, de obtener mayor información. Además, la instalación de una red interna dentro de la empresa supone la reconciliación de cuatro mundos muy diferentes: sistemas de información y bases de datos, documentación técnica (planes, software), comunicación (correo electrónico, etc.) y el mundo exterior.

Los sitios Intranet son más fáciles de gestionar que los sitios Internet. Una de las reglas básicas para la gestión de un sitio Intranet de Web es que deberían renovarse y cambiar el sitio para que los usuarios lo sigan utilizando. Si navegan sin problemas serán más propensos a volver.

El diseño de su sitio debe tener en cuenta a sus usuarios: es necesario que sea suficientemente fácil de usar y entretenido para mantenerlos satisfechos. El administrador de un sitio Intranet (el webmaster) promociona crecimiento, acceso, hospitalidad, eficiencia, organización y utilidad.

Una de las mejores formas para controlar la información corporativa es a través de una Intranet. Para que las Intranets basadas en tecnologías Web sean útiles y valiosas para los usuarios, deben ir más allá de la pura difusión de información y proporcionar recuperación y gestión flexible de casi toda la información de la empresa. Las plantillas de los departamentos de sistemas de 
información también deben adaptarse a una nueva generación de aplicaciones de empresa basadas en el Web. Todavía no existen datos concretos que muestren la implantación y adaptación de tecnología Intranet en empresas españolas, ${ }^{7}$ pero sí han señalado un alto grado de aceptación y satisfacción por la nueva forma de generar y organizar la información dentro de la empresa.

Un sitio Web en una Intranet sirve para un propósito concreto. El webmaster tiene que comprender y conocer la audiencia de su sitio de Web, y periódicamente evaluarlo e identificar cuáles son sus necesidades, qué es lo que quieren los usuarios y cómo pueden utilizar el sitio de otros modos. Su función de controlador de la circulación y generación de la información dentro de la empresa es responsabilidad suprema, lo que constituye un punto a tener en cuenta si realmente pensamos quién es el que lo controla.

Un aspecto a valorar es la actitud de los trabajadores ante el cambio. El hecho de que se conviertan en miembros activos de una red cooperativa influye positivamente en la marcha de la empresa, traducida en productividad y buena comunicación interna.

Una aplicación de comunicación obvia de la era Internet que se hace imprescindible en una Intranet, es además del correo electrónico, las populares conexiones de chat . Éstas han dado lugar rápidamente a las salas de conferencia en línea, donde los socios pueden conocer y compartir sus respectivos planes y datos. Un beneficio añadido a la sala de conferencias on line es la capacidad de archivar discusiones para posteriores referencias. Pero la tecnología de videoconferencia a través de una Intranet, presenta la dificultad del ancho de banda. La señal digital ocupa gran cantidad de ancho de banda, lo que por el momento obliga a realizar la videoconferencia por medio de video por satélite.

El futuro está en el diseño de herramientas para modificar internamente la Intranet y reestructurarla fácilmente, aumentar su

7 Según una encuesta realizada a las pymes españolas por la Fundación Cotec para la Innovación Tecnológica, el motivo fundamental por el que invierten en tecnología es para la mejora de la calidad global de la empresa (50\%) y le sigue a gran distancia el ahorro de tiempo (26\%) junto con la mejora del control del negocio (22\%). 
flexibilidad, añadir más plug-ins y aplicaciones gráficas o multimedia, y otros productos para su fácil adaptación al Web.

Quizá lo que más ha cautivado de la red intranet es, según Shel Holtz (1996: 54-57), el can y no el will, es decir, una realidad presente en nuestras empresas y no unas promesas tecnológicas difíciles de cumplir.

\section{Bibliografía}

Cruz, Fernando (1997), "Intranets seguras para las empresas", en Expansión, febrero.

Edmonson, Gail (1996), "Here comes the intranet", en Bussines Week, february.

Fryer, Bronwyn (1996), "Intranetmania" en Datamation, núm. 125.

García Bravo, Daniel (1995), Sistemas y tecnologías de la información en la empresa, Alicante: Instituto de Cultura Juan Albert,.

Holtz, Shel (1996), "Intranet" en Communication World, June/July.

Huidrobo, José Manuel. (1996), “Redes corporativas: ¿qué y cómo?”, en Datamation, marzo.

Lefebvre, Alain (1997) ," Intranet. Cliente-servidor universal” en Gestión 2000, Barcelona.

(1997), "Conozca su red interna: Intranet e Internet. Informática para su empresa", en Digital, Madrid.

Lezaun, Jean-Louis (1997), "Las teconologías que revolucionan la empresa”, ponencia en XIV Congreso de Estudios Vascos, Bilbao-Madrid, 25-27 noviembre.

Molina, Paula (1997), "Intranets, comunicaciones seguras en la empresa", en Super Net Magazine, núm. 19

S/A (1996), "Una intranet en cada empresa", en Expansión, 12 de julio.

Navas López, J.E. (1994), “Organización de la empresa y nuevas tecnologías”, en Pirámide, Madrid.

Parrilla, J.C (1996), "Redes de área local”, en Síntesis, Madrid.

Sánchez Crespo, Daniel (1996), "Internet en la empresa Guía profesional”, en Inforbook's. Barcelona.

Shamon, David (1997), "Intranets y los presupuestos de SI", en Datamation, enero.

Tuñon, D (1997), “ Intranets, las redes más corporativas”, en Net Conexion, núm. 16. 